\title{
The adult component of selection in Drosophila melanogaster: some aspects of early-remating activity of females
}

\author{
A. VAN VIANEN \& R. BIJLSMA* \\ Department of Genetics, University of Groningen, Kerklaan 30, 9751 NN Haren (GN), The Netherlands
}

\begin{abstract}
As an important factor of the adult component of selection, mating behaviour was studied in Drosophila melanogaster, with emphasis on non-virgin females. We found that 30-50 per cent of the females in a laboratory population will remate within $6 \mathrm{~h}$ of first mating under no-choice conditions. This high percentage of early rematings was not due to the continuous confinement of the females with males but indicated a rapid return of receptivity of a significant proportion of the females. Remating behaviour was significantly influenced by both the genotype of the female and the genotype of her two successive partners. Age of females was only important insofar as it concerned young, 1 or 2 -day old, females. These females showed less remating than older females. Willingness to remate was also affected by the number of sperm stored. Females that had been inseminated by less fertile males, i.e. males that had already mated two or three times, showed higher remating percentages than females inseminated by more fertile males. Notwithstanding this sperm effect, females were estimated to remate approximately every second day. It is suggested that a high frequency of remating and the resulting sperm competition are significant components of Drosophila life-history.
\end{abstract}

Keywords: adult selection, Drosophila melanogaster, mating behaviour, remating, sperm competition.

\section{Introduction}

The adult component of selection is, besides viability selection in the larval stage, an important component of selection in Drosophila species (Prout, 1971a,b; Bundgaard \& Christiansen, 1972; Bijlsma-Meeles \& Bijlsma, 1988). In particular, components related to mating and fertility can be significant with respect to the maintenance of polymorphisms (Hedrick \& Murray, 1983).

An important aspect in this context is that $D$. melanogaster females may mate more than once during their lifetime. Multiple mating of females is common in insects. Insect females often have organs specialized for sperm storage (Parker, 1970). In D. melanogaster, remating of females which have sperm from previous matings left in storage, results in sperm competition and most often sperm from the last male take precedence over those of previous male(s) and preferentially fertilize subsequent eggs (Prout \& Bundgaard, 1977). This process of sperm displacement generally leads to

*Correspondence. sperm loss of the previous male(s). This loss increases considerably as the number of sperm of prior mating( $\mathrm{s})$ in storage increases, and when the time lapse between two successive matings becomes shorter (Prout \& Bundgaard, 1977; Scott \& Richmond, 1990). The consequences of remating, therefore, depend highly upon how frequently females remate. The objective of this paper is to evaluate the opportunity for sperm competition to occur by studying remating, in particular early rematings (rematings that occur within a few hours after a previous mating), and some factors that may affect the frequency of remating.

Some laboratory studies have reported that females become very reluctant to remate after a successful copulation (Manning, 1962; Gromko et al., 1984; Letsinger \& Gromko, 1985; Pyle \& Gromko, 1981). These authors postulated two mechanisms that prevent an already inseminated female from remating: a shortlasting copulation effect and a long-lasting sperm effect. Unreceptivity due to the copulation effect persists for about one day and may be a response to both the act of copulation itself and to components of the seminal fluids transferred from males to females 
during copulation (Chen, 1984; Scott, 1987). Of these substances in the seminal fluid, a specific sex-peptide seems to be responsible for both induction of unreceptivity in females, as well as for stimulation of oviposition in D. melanogaster (Chen, 1984; Chen et al., 1988). The basis for the sperm effect is the amount of sperm that a female has stored. When this amount is still above a certain threshold level the female will not remate. Gromko and coworkers have estimated that under natural conditions females will mate every 4th or 5th day, after the sperm of the previous mating has been depleted by more than 80 per cent. They argue therefore, that competition between sperm of different males for fertilization of eggs cannot be an important selective force.

Gromko and coworkers used an experimental approach in which the females only had access to males for 2 hours every day, because they suggested that this approach is more representative of mating behaviour of D. melanogaster in the field (but see discussion in Harsham et al., 1988). When females have access to males continuously for $24 \mathrm{~h}$, however, remating frequencies of 40-80 per cent have been observed (Prout \& Bundgaard, 1977; Newport \& Gromko, 1984) indicating that the sperm effect may not be strong enough to prevent the occurrence of significant levels of sperm competition. Moreover, Fukui \& Gromko (1989) showed that females taken from natural populations show a less pronounced sperm effect and are willing to remate more readily after their first mating than females from laboratory strains. These latter observations agree well with the high incidence of multiple inseminations (25-60 per cent of wild-caught females have been estimated to produce offspring of at least two different males), that has been observed in natural populations of D. melanogaster (e.g. Milkman \& Zeitler, 1974; Griffith et al., 1982; Marks et al., 1988) suggesting that females often remate and maintain a high level of sperm in storage possibly to maximize their life-time-progeny production.

The copulation effect also may be less effective than thought. Some authors (Fuerst et al., 1973; Bundgaard \& Christiansen, 1972) observed that some females (about 10-25 per cent) already remate within a few hours of the first mating. Scott (1987) examined this effect more closely and reported even higher levels of early rematings.

In this paper we have analysed some aspects of the remating process of $D$. melanogaster females in order to answer the following questions: (i) how fast and to what extent do females remate after their first mating? (ii) Are there genetic differences between strains with respect to remating activity? (iii) How do a number of non-genetic factors like female reluctance, age of the flies and sperm depletion of males influence the remating process?

\section{Materials and methods}

\section{Strains}

Three wild-type laboratory strains of $D$. melanogaster were used: (i) Bogota, collected in Colombia in 1965 and maintained since then in mass culture in a population cage; (ii) Groningen 1983 (further referred to as G83) collected at the Groningen Fruit Auction in 1983 and kept in the same way as the strain Bogota; (iii) Groningen 1987 (G87), collected at the same fruit auction but in 1987 .

During experiments offshoots of these strains were maintained in five half pint bottles per strain and the flies were regularly (1-5 times a week) transferred to new bottles for egg laying to ease virgin collecting. To avoid divergence between the bottles, within each strain flies from the five bottles were from time to time mixed.

All strains were reared on standard food (Bijlsma \& Van Delden, 1977$)$ at $25^{\circ} \mathrm{C}, 40-60$ per cent r.h. and under a light/dark cycle of $16 / 8 \mathrm{~h}$. Experiments were carried out at the same regime on standard food to which $100 \mathrm{mg} \mathrm{l}^{-1}$ streptomycin was added to prevent excessive bacterial growth. All flies used were collected as virgins, within $6 \mathrm{~h}$ of eclosion, without anaesthesia. Flies used in the experiments were 4-6 days old, unless stated otherwise.

\section{Influences of genotype on mating and remating}

A male (male-1) and a female were brought together in a vial and given $50 \mathrm{~min}$ to start copulation. Immediately after termination of the copulation the male was discarded and the female was provided with a new virgin male (male-2). This pair was then observed at 10-min intervals to see whether copulation occurred, for up to $6 \mathrm{~h}$. In this way females of all three strains were provided with two successive males of their own strain. Furthermore, to test whether or not strain differences affected the remating activity of females for the strains Bogota and G83, all eight possible combinations of females and the two consecutive males were tested.

Additionally, for Bogota females only, the experiment was repeated in a slightly different way. In this case the female was not provided with the second male directly after termination of the first copulation but was kept isolated for $3 \mathrm{~h}$. After this period male- 2 was introduced and the vials were observed for remating for the next $3 \mathrm{~h}$. 
All mating experiments were initially started with 80-120 pairs of flies for each combination. Depending on the number that actually mated, remating tests were done with 50-100 pairs per combination.

\section{Influence of some intrinsic factors on remating}

Female reluctance. It is conceivable that the remating activity of a female depends on an intrinsically fixed level of mating reluctance and that females that are reluctant to mate for their first time, when still virgins, are also reluctant to remate. This was tested for the G87 strain only. Single pairs were allowed to mate for $1 \mathrm{~h}$ and the copulation latency time (CLT1, the time lapse between introduction of the male- 1 and the beginning of the copulation) was recorded. After the first copulation was terminated, male- 2 was introduced and for this male CLT 2 was recorded, for up to $6 \mathrm{~h}$.

Age. To examine the effect of age of flies on remating activity, three different experimental designs were used:

(i) Females were aged as virgins for the necessary number of days and then provided consecutively with two 4 or 5-day-old males of the same strain and monitored for remating with the second male for a period up to $6 \mathrm{~h}$. This was done for Bogota females (aged 1-7 days) and G83 females (aged 1-10 days).

(ii) Three virgin females and three males were placed in a vial within $1 \mathrm{~h}$ of eclosion. This was done for 10 consecutive days and in this way a series of 20-25 vials was obtained containing three pairs of flies that were housed together for $0-10$ days, giving them ample opportunity to mate and remate. Consequently the flies were 0-10 days old, and within each vial all flies were the same age. On the next day, day 11 , all vials were observed for matings during the whole 16-h-light period. This experiment was done twice, for the strain G83.

(iii) To separate the effect of the difference in age from the difference in the length of the period the flies were able to mate, the experiment was modified such that all flies observed were the same age. They were all collected on the starting day of the experiment, but kept separated for 0-10 days thereafter. Each day a series of vials was prepared that contained three pairs of flies, as described above. In this way a series of vials was obtained that contained flies that differed only in the period that they were given the opportunity to (re)mate but not in age. On the 10th day of the experiment observations as described above were made. In addition, on this day also a series of vials was prepared containing virgin flies. After an initial period of mating activity this series was monitored for remating. This experiment was done once for the G83 strain only.
Mating history of the male. Single 5-day-old G83 males were allowed to mate with four G83 females of the same age consecutively. In this way four different types of females that received sperm from males with a different mating history were created: type- 1 females were mated to virgin males, type- 2 females to males that had previously mated once, etc. Twenty complete series of four females mated consecutively to the same male were allowed to lay eggs and their total number of progeny was counted.

Another group of females, representing the same four types, but not necessarily inseminated by the same male, was given the opportunity to remate with a 5-day-old virgin male for $1 \mathrm{~h} 5$ days after the initial copulation. For each female type the remating percentage was established. For comparison the mating percentage of virgin 10-day-old females to virgin 5-day-old males was also measured.

\section{Results}

\section{Genotype differences}

Females from all three strains showed high percentages of remating (30-50 per cent) with males of the same strain within $6 \mathrm{~h}$ of their first mating (Fig. 1). The total remating percentage after $6 \mathrm{~h}$ was significantly higher for the strain Bogota than for the strain G87 (contingency $\chi^{2}=7.2 ;$ d.f. $=1 ; P<0.05$ ), while the total remating percentage of the strain G83 was in between the other two strains and did not differ significantly from either of them $(P>0.05)$.

The steady increase in the number of rematings during the 6-h observation period as shown in Fig. 1 may be either due to the fact that in the course of time more and more females become receptive again after their first mating or due to the continuous sexual 'harassment' by the males from which the females cannot escape in the vials. Figure 2 shows that, when the females were not combined with the second male directly after the first mating (broken lines) but kept separate for $3 \mathrm{~h}$ after this mating (solid lines) the cumulative number of rematings increases significantly faster. The group of females that did not get the opportunity to remate during the first $3 \mathrm{~h}$ reached around 40 per cent of remating within $2 \mathrm{~h}$ after the second male was provided, while it took the other females $4-5 \mathrm{~h}$ to reach this level. This indicates that the return of receptivity is not (solely) due to the prolonged attendance of persistent males but that it is actually due to an intrinsic change in the females.

When combined with different sequences of the two consecutive males, Bogota and G83 females did not differ significantly in remating performance. Averaged 
over the different male combinations, 52.1 per cent of the G83 females had remated after $6 \mathrm{~h}$ and of the Bogota females 51.0 per cent (Table 1). However, within the strain Bogota there were significant differ-

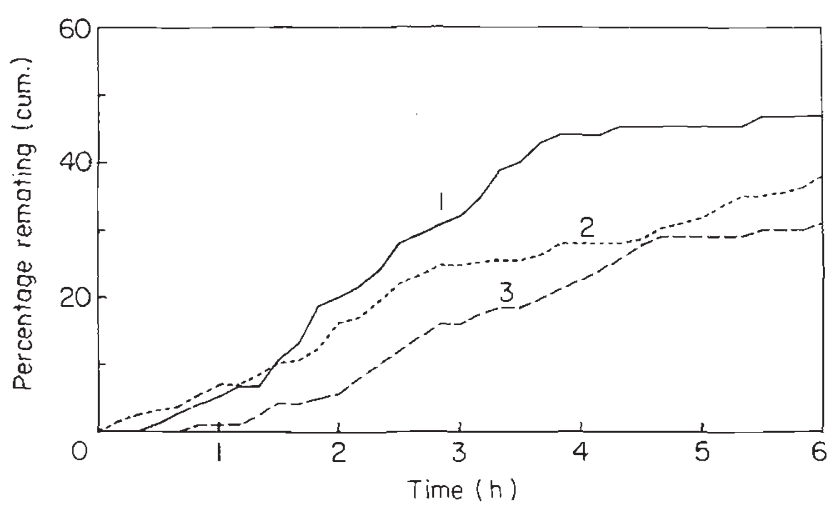

Fig. 1 Cumulative remating percentages of females with males of the same strain during a 6 -h period for three wildtype strains. (1) Bogota $(n=282),(2)$ Groningen 1983 $(n=368),\{3)$ Groningen $1987(n=120)$. $(n=$ number of females tested.)

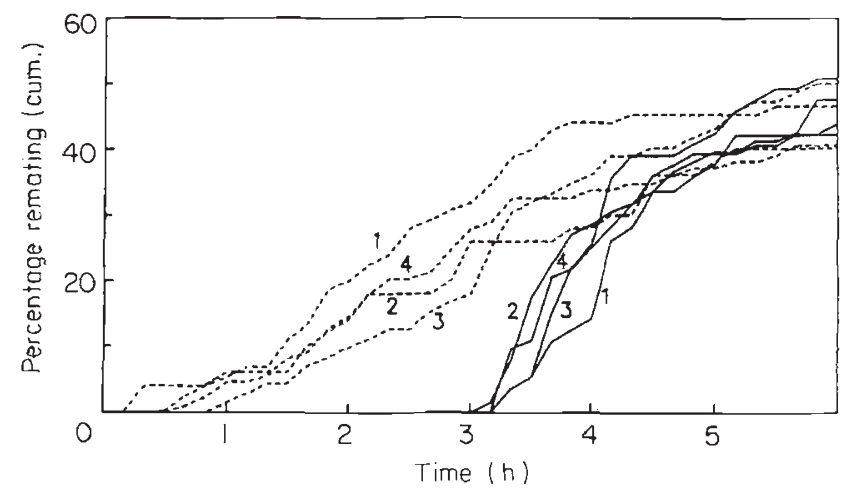

Fig. 2 Comparison of two types of remating experiments in which the female was either provided with a second male directly after copulation (broken lines) or $3 \mathrm{~h}$ after copulation (solid lines). Different female-male-male combinations were tested: (1) BBB, (2) BGB, (3) BBG and (4) BGG. For the meaning of the letter combinations see Table 1 . ences (contingency $\chi^{2}=11.06$; d.f. $=3 ; P<0.05$ ) with respect to the different male combinations, but no significant differences were observed for G83 females (contingency $\chi^{2}=3.32$; d.f. $=3 ; P>0.30$ ). By pairwise comparison of the different male combinations, the effect of male genotype on remating can be examined. Comparing the different combinations that differed with respect to the second male (BBB-BBG, BGB-BGG, GBB-GBG and GGB-GGG; for an explanation of the letter combination see legend of Table 1), we can see that the percentage of remating was always higher, though not significantly (contingency $\chi^{2}=1.19 ;$ d.f. $=3 ; P>0.15$ ), for the G83 males. The influence of the first male can be examined by comparing combinations that differed for male- 1 but were the same for male-2 (BBB-BGB, BBG-BGG, GBB-GGB and GBG-GGG). All four comparisons of females that were mated to G83 as male-1 showed significantly lower remating percentages than when mated to Bogota males as male-1 (contingency $\chi^{2}=7.15$; d.f. $=3 ; P>0.01$ ). Both remating with nonvirgin females and restraining females from remating with other males are important components of male fitness. In this sense G83 males were better competitors and outperformed Bogota males in all eight pairwise comparisons (sign test; $P<0.01$ ).

\section{Effect of some intrinsic factors}

Female reluctance. No significant differences in remating activity due to possible differences in intrinsic reluctance levels of the females were observed. Both fast-mating females $(n=72)$ that mated within the first 30 min after the male was introduced in the vial and slow-mating females $(n=36)$ that mated in the second 30 min of the experiment showed about 30 per cent remating. The observed difference was not significant $(P>0.05)$. Also no significant correlation between the latency times of first and second mating were observed (Spearman rank correlation test, $r_{s}=-0.20, P>0.05$ ).

Table 1 Remating percentages within $6 \mathrm{~h}$ of Bogota $(\mathrm{B})$ and Groningen $83(\mathrm{G})$ females with different combinations of consecutive male genotypes: the first letter denotes the female genotype, the second letter the first male and the third letter the second male. ( $n=$ number of pairs tested)

\begin{tabular}{|c|c|c|c|c|c|}
\hline \multicolumn{3}{|c|}{ Bogota females } & \multicolumn{3}{|c|}{ Groningen females } \\
\hline Combination & $\%$ Remating & $n$ & Combination & $\%$ Remating & $n$ \\
\hline $\mathrm{BBB}$ & 56.0 & 75 & GBB & 51.4 & 74 \\
\hline $\mathrm{BBG}$ & 66.7 & 72 & GBG & 57.4 & 61 \\
\hline BGB & 42.0 & 50 & GGB & 42.0 & 62 \\
\hline BGG & 43.8 & 89 & GGG & 53.5 & 129 \\
\hline
\end{tabular}




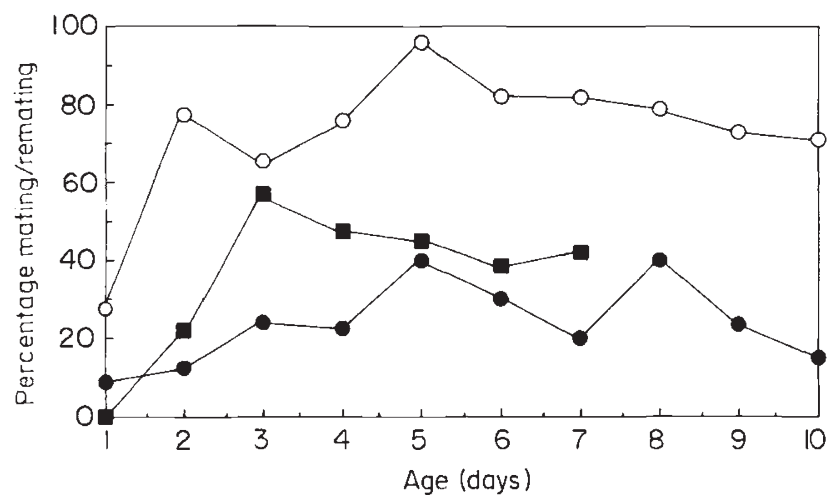

Fig. 3 Influence of age on mating and remating for females of two different strains. (O) Mating percentage of virgin Groningen 83 females within 50 min, remating percentages of (•) Groningen 83 and (-) Bogota females during $6 \mathrm{~h}$. Females of all ages were mated to males 4-5 days old.

Age. Figure 3 shows that virgin females mated readily when they were combined with a 4-5-day-old virgin male, except when the females were 1 day old. Only 30-40 per cent of these females mated within the 50min observation period. Additionally, the 2-3-day-old females showed a somewhat, but not significantly, lower mating percentage than the older females of which around 80 per cent mated within the observation period. Remating of females seems to be influenced by age in a similar way (Fig. 3). Very young flies showed low levels of remating within $6 \mathrm{~h}$ of copulation (e.g. 10 per cent for 1-day-old flies). Remating percentages of 3 day-old and older females showed fluctuations between 20 and 40 per cent for the $\mathrm{G} 83$ females, while the Bogota females showed levels of remating up to 50 per cent for 3-5-day-old individuals. This higher level of remating of Bogota females compared to G83 females agreed well with the difference observed in Fig. 1.

Figure 4(a) shows the results of the experiment in which vials with three pairs of flies (G83) were observed during the 16-h light period. Except for considerable fluctuations (the high frequency of matings observed at day 1 is explained by the fact that it involves both mating of virgin females and rematings) during the first few days, the number of (re)matings seemed fairly constant and not dependent on age and housing period within each of the two replicated tests. The two replications showed a somewhat different level of remating, but no consistent correlation between the age of the flies and the level of remating was found in either (Spearman Rank correlation test: $r_{\mathrm{s}}=-0.39, P>0.05$ and $r_{\mathrm{s}}=0.35, P>0.05$ for experiment 1 and 2 , respectively). The period that the three pairs of flies had been housed together in one vial (Fig. $4 b)$ seemed to have no significant effect on the number

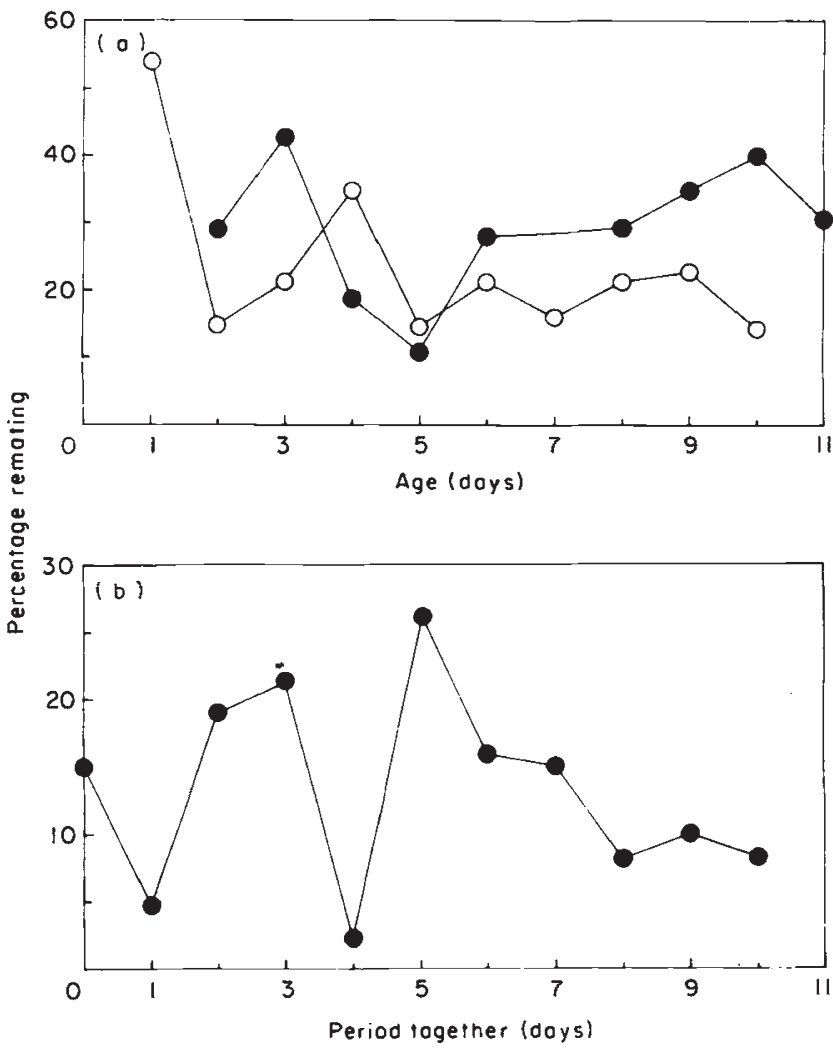

Fig. 4 Matings observed during a 16-h light-period experiment for Groningen 83. (a) Flies 1-11 days old; females and males combined in vials shortly after eclosion. The results of two replicated experiments are shown. (b) Flies all 10 days old, but males and females combined for 0-10 days before the observation period.

of (re)matings $\left(r_{\mathrm{s}}=-0.13, P>0.05\right)$, although there appears to be a tendency for an optimum around day 5 . The average number of matings observed per vial (excluding age 0 and 1 day) was $0.87 \pm 1.03$. On average $20-30$ per cent of the females remated during the 16-h light period.

Mating history of the male. Table 2 shows the progeny numbers and remating percentages of the different female types. Females mated to virgin males or to males that had mated only once before (type- 1 and type- 2 females, respectively) produced significantly higher numbers of offspring than females of type- 3 and type4. Females of type-4, which were mated to males that already had performed three matings in short succession before their fourth mating, showed a particularly marked reduction in offspring of almost 50 per cent compared to females of type- 1 and type- 2 . This indicates that females of type- 3 and type- 4 have had lower fertilization levels, as expected, due to lower amounts of, or less effective, sperm transferred to them than females of type- 1 and type- 2 . 
Table 2 Mean offspring numbers $( \pm$ S.E.) produced by the different female types together with the remaining percentages of these females. The number of replicates for each test is shown in brackets. Means or percentages marked by different letters differ significantly from each other $(P<0.05)$

\begin{tabular}{lcl}
\hline Female type & Progeny numbers & Percentage $($ re)mating \\
\hline 1 & $107.6 \pm 12.5 \mathrm{a}(20)$ & $40.0 \mathrm{a}(60)$ \\
2 & $108.7 \pm 11.9 \mathrm{a}(20)$ & $41.9 \mathrm{a}(43)$ \\
3 & $85.0 \pm 12.7 \mathrm{~b}(20)$ & $72.5 \mathrm{~b}(40)$ \\
4 & $56.3 \pm 9.7 \mathrm{c}(20)$ & $86.2 \mathrm{~b}, \mathrm{c}(29)$ \\
virgins & - & $92.1 \mathrm{c}(60)$ \\
$(10$-day-old) & & \\
\hline
\end{tabular}

In remating tests, females of type- 1 and type- 2 were more reluctant to remate than females of type- 3 and type- 4 , but still showed remating percentages around 40 per cent within $1 \mathrm{~h}$. This was, however, significantly lower than the remating percentages found for females of type- 3 and type- 4 . Females of type- 4 showed the same amount of mating as virgin females of the same age, indicating that they had returned to full receptivity.

\section{Conclusions and discussion}

Remating patterns (Figs 1 and 2) of all strains showed similar characteristics. Directly after copulation, remating percentages were low but increased rapidly $1-4 \mathrm{~h}$ after copulation up to $30-50$ per cent, depending on the strain. Thereafter this increase levelled off. An analysis of the pattern with statistics designed for truncated samples (Bliss, 1967; Dow, 1978) indicated that longer observation times would not have given significantly higher remating levels than those recorded after $6 \mathrm{~h}$. The observed high remating percentages are in line with results obtained by Scott (1987), who found that 15-30 per cent of the females remated between 4 and $5 \mathrm{~h}$ after the first copulation and 30-40 per cent between 6 and 7 h. Scott (1987) has shown that within the 6-h period after mating the presence of sperm stored in the female does not cause a decrease in receptivity beyond that due to the act of copulation and the transfer of seminal fluid only. The sharp decrease in receptivity of the females should therefore be explained in terms of the copulation effect. The return to receptivity might possibly be governed by some of the substances (e.g. esterase-6) present in the seminal fluid of the male, which are transferred to the reproductive tract of the female and can be found in the haemolymph within a few hours of copulation (Richmond \& Senior, 1981; Meikle et al., 1990). But courtship-inhibiting pheromones that are transferred from males to females during copulation and that gradually decrease in concentration thereafter may also play a role (Scott \& Jackson, 1988). The onset of oviposition in D. melanogaster falls between 4 and $6 \mathrm{~h}$ after mating (Scott \& Richmond, 1990) and this may be (partly) responsible for the decrease in remating activity during this period as it decreases receptivity in many insect species (Giebultowicz et al., 1990).

In our remating experiments females were continuously confined with males and, without the possibility for the females to decamp, this procedure may lead to unusually high remating frequencies (Newport \& Gromko, 1984). However, we did not observe a difference in the final remating frequencies whether females were confined with males directly after the first copulation or kept isolated during the first $3 \mathrm{~h}$ (Fig. 2). This indicates that the increase in receptiveness of females to remating is the result of an intrinsic process and not 'forced' by the presence of the males. Furthermore, Partridge et al. (1987) observed that decamping by $D$. melanogaster females is not the main cause for termination of courtship in the field. We think, therefore, that the observed high remating frequencies are not the consequence of the experimental procedure and that a significant proportion of the females may remate within a few hours of a prior mating.

Remating frequencies seem not to be affected by age of the females except when they are young (1-2 days old; Fig. 3). These young females in general appear reluctant to mate because both the frequency of first matings and of rematings are lower at this age. This apparent correlation between reluctance of females and remating activity, however, was not observed for older females, aged 4-6 days.

The frequency of early rematings is clearly strain dependent (Figs 1 and 2) and is also affected by the genotype of the males involved (Table 1). G83 males seemed better competitors than Bogota males. They were both more successful in mating with non-virgin females and in restraining females mated to them from remating with another male. We did not find significant differences in body size, fertility or male-male competition between the strains that could explain the observed differences. Eastwood \& Burnet (1977) have shown that males may differ genetically in their readiness to court non-virgin females. This may explain the difference in ability to induce females to remate but seems not to account for the difference observed in remating frequencies of the females mated to them. The latter is more likely caused by differences in the amount or quality of the seminal fluid transferred during copulation. Furthermore, differences between the females might also be important in this respect.

According to Scott (1987) the sperm effect becomes evident $8-12 \mathrm{~h}$ after mating, causing complete 
unreceptivity of females thereafter. Return to receptivity occurs again when sperm becomes depleted and the amount of sperm in storage decreases below a threshold level (Gromko et al., 1984). Our experiment on the mating history of the males seems to confirm these findings. Females that were inseminated by males that already had spent most of their sperm in prior matings were the most willing to remate. These females also produced fewer progeny than females inseminated by males that had not mated previously. Obviously, the first group of females suffered from sperm depletion and therefore was more receptive. A similar result was observed by Markov et al. (1978), who showed that male fertility was significantly reduced at the third consecutive mating. The data presented in Fig. 4 seem to contradict this effect. In these experiments one would expect an increase in the number of rematings when the time interval that the flies were housed together increases, due to the depletion of the number of sperm stored after the initial matings. However, the number of rematings seems to be fairly constant; at least, there is no consistent positive relation between remating percentage and time after the initial mating. Furthermore, the level of remating within $1 \mathrm{~h}$ after introduction of a virgin male is the same in females that are isolated for $3 \mathrm{~h}$ (Fig. 2) or for 5 days (Table 1, type-1 females) after their initial copulation (40 per cent). This suggests that a more or less constant fraction of previously inseminated females, irrespective of the level of sperm depletion, is willing to remate, despite copulation and sperm effect. From our observations presented in Figs 3 and 4 it can be deduced that females will remate approximately every $2-3$ days. This number is probably an underestimation because we only recorded the rematings that occurred during the light period. Some limited observations during the dark period indicated that females also remate during the dark period. We estimate, therefore, that females probably mate once every day or second day. This estimate agrees with the data given by Bellen \& Kiger (1987) who continuously observed vials with three males and one female for 1 week. They found that Canton-S females in this situation mated every $22-24 \mathrm{~h}$. In natural populations remating might also be frequent. Marks et al. (1988) estimated by different methods the frequency of multiple inseminations in two distinct habitats outside and inside a winery building (flies were not resident inside the winery but accumulated there during daytime). In contrast to the outside females, females inside the winery revealed a high frequency of multiple inseminations (12 vs. 65 per cent), and Marks et al. (1988) infer that perhaps the greater number of these females had remated within the last $12 \mathrm{~h}$.
Notwithstanding the existence of both copulation and sperm effects, our results indicate that these mechanisms are not sufficient to prevent substantial levels of remating. These levels, however, are significantly higher than those reported by Gromko and coworkers (Gromko et al., 1984; Letsinger \& Gromko, 1985; Pyle \& Gromko, 1981). Given the results of the early remating experiment, this difference seems not to be explained by the continuous confinement procedure we used. Moreover Fukui \& Gromko (1989) recently reported similar levels of remating for freshly caught females. The finding that females remate often is more in line with the high number of multiple inseminations found in natural populations (e.g. Milkman \& Zeitler, 1974; Marks et al., 1988). This higher frequency of remating implies that females will remate when they still have a considerable amount of sperm of previous matings in storage and consequently increased sperm competition will result (Prout \& Bundgaard, 1977; Fukui \& Gromko, 1989; Scott \& Richmond, 1990). Therefore we conclude that remating, and the resulting sperm competition, are not marginal processes but significant components of Drosophila life-history.

\section{Acknowledgements}

We thank W. van Delden, J. Bundgaard, and two anonymous reviewers for their critical comments. Albert Kamping provided strain G83 and was helpful in collecting strain G87. Jettie Sismanoglu, Laurence Hoeksema-du Pui and Jan Graffelman prepared the media. We thank Henk Mulder for drawing the figures.

\section{References}

BELLEN, H. J. AND KIGER, J. A. 1987. Sexual hyperactivity and reduced longevity of dunce females of Drosophila melanogaster. Genetics, 115, 153-160.

BIJLSMA, R. AND VAN DELDEN, w. 1977. Polymorphism at the G6PD and 6PGD loci in Drosophila melanogaster. I. Evidence for selection in experimental populations. Genet. Res., 30, 221-236.

BIJLSMA-MEELES, E. AND BIJLSMA, R. 1988. The alcohol dehydrogenase polymorphism in Drosophila melanogaster, fitness measurements and predictions under conditions with no alcohol stress. Genetics, 120, 743-753.

Buss, C. J. 1967. Statistics in Biology, vol. 1. McGraw-Hill, New York, pp. 162-167.

BUNDGAARD, J. AND CHRISTIANSEN, F. B. 1972. Dynamics of polymorphism: I. Selection components in an experimental population of Drosophila melanogaster. Genetics, 71, 439-460.

CHEN, P. S. 1984. The functional morphology and biochemistry of insect male accessory glands and their secretions. Annu. Rev. Entomol., 29, 233-255. 
CHEN, P. S., STUMM-ZOLLINGER, E., AIGAKI, T., BALMER, J., BIENZ, M. AND BOHLEN, P. 1988. A male accessory gland peptide that regulates reproductive behavior of female $D$. melanogaster. Cell, 54, 291-298.

Dow, M. A. 1978. Analysis of truncated distributions: mating speed in Drosophila melanogaster. Behav. Genet., 6, 385-389.

EASTwOOD, L. AND BURnET, B. 1977. Courtship latency in male Drosophila melanogaster. Behav. Genet., 7, 359-372.

FUerst, P. A., PEndlebury, w. W. AND KidWell, J. F. 1973. Propensity for multiple mating in Drosophila melanogaster females. Evolution, 27, 265-268.

FUKUI, H. H. AND GROMKO, M. H. 1988. Female receptivity to remating and early fecundity in Drosophila melanogaster. Evolution, 43, 1311-1315.

GIEBULTOWicz, J. M., RAINA, A. K. AND UEBEL, E. C. 1990. Matedlike behaviour in senescent virgin females of gypsy moth, Lymantria dispar. J. Insect Physiol., 36, 495-498.

GRIFFITH, R. C., MCKECHNIE, S. W. AND MCKENZIE, J. A. 1982. Multiple mating and sperm displacement in a naturál population of Drosophila melanogaster. Theor. Appl. Genet., 68, 89-96.

GROMKO, M. H., NEWPORT, M. E. A. AND KORTIER, M. G. 1984. Sperm dependence of female receptivity to remating in Drosophila melanogaster. Evolution, 38, 1273-1282.

HARSHMAN, L. G., HOFFMANN A. A. AND PROUT, T. 1988. Environmental effects on remating in Drosophila melanogaster. Evolution, 42, 312-321.

HEDRICK, P. W. AND MURRAY, E. 1983. Selection and measures of fitness. In: Thompson, J. and Ashburner, M. (eds) Genetics and Biology of Drosophila, vol. 3, Academic Press, New York, pp. 61-104.

LETSINGER, J. T. AND GROMKO, M. H. 1985. The role of sperm numbers in sperm competition and female remating in Drosophila melanogaster. Genetica, 66, 195-202.

MANNING, A. 1962. A sperm factor affecting the receptivity of Drosophila melanogaster females. Nature, 194, 252-253.

MARKOV, T. A., QUAID, M. AND KERR, s. 1978. Male mating experience and competitive courtship success in Drosophila melanogaster. Nature, 276, 821-822.

MARKS, R. W., SEAGER, R. D. AND BARR, L. G. 1988. Local ecology and multiple mating in a natural population of Drosophila melanogaster. Am. Nat., 131, 918-923.
MEIKLE, D. B., SHEENAN, K. B., PHILLIS, D. M. AND RICHMOND, R. C. 1990. Localization and longevity of seminal-fluid esterase-6 in mated female Drosophila melanogaster. J. Insect Physiol., 36, 93-101.

MILKMAN, R. AND ZEITLER, R. R. 1974. Concurrent multiple paternity in natural and laboratory populations of Drosophila melanogaster. Genetics, 78, 1191-1193.

NEWPORT, M. E. A. AND GROMKO, M. H. 1984. The effect of experimental design on female receptivity to remating and its impact on reproductive success in Drosophila melanogaster. Evolution, 38, 1261-1272.

PARKER, G. A. 1970. Sperm competition and its evolutionary consequences in insects. Biol. Rev., 45, 525-567.

PARTRIDGE, L., HOFFMANN, A. A. AND JONES, S. J. 1987. Male size and mating success in Drosophila melanogaster and $D$. pseudoobscura under field conditions. Anim. Behav., 35, 468-476.

PROUT, T. 1971a. The relation between fitness components and population prediction in Drosophila I: The estimation of fitness components. Genetics, 68, 127-149.

PROUT, T. $1971 \mathrm{~b}$. The relation between fitness components and population prediction in Drosophila II: Population prediction. Genetics, 68, 151-167.

PROUT, T. AND BUNDGAARD, J. 1977. The population genetics of sperm displacement. Genetics, 85, 95-124.

PYLE, D. W. AND GROMKO, M. H. 1981. Genetic basis for repeated mating in Drosophila melanogaster. Am. Nat., 117, $133-146$.

RICHMOND, R. C. AND SENIOR, A. 1981. Esterase-6 of Drosophila melanogaster: kinetics of transfer to females, decay in females and male recovery. $J$. Insect Physiol., 27, 849-859.

SCOTT, D. 1987. The timing of the sperm effect on female Drosophila melanogaster receptivity. Anim. Behav., 35, 142-149.

SCOTT, D. AND JACKSON, L. L. 1988. Interstrain comparison of male-predominant antiaphrodisiacs in Drosophila melanogaster. J. Insect. Physiol., 34, 863-871.

SCOTT, D. AND RICHMOND, R. C. 1990. Sperm loss by remating Drosophila melanogaster females. J. Insect Physiol., 36, 451-456. 